\title{
Improving the accuracy of manufacturing long shafts and axes of precast rotors
}

\author{
Mikhail Popov ${ }^{1, *}$, Andrei Popov ${ }^{1}$, and Luai Al-Obaidi ${ }^{1}$ \\ ${ }^{1}$ Don State Technical University, sq. Gagarina, 1, Rostov-on-Don, 344010, Russia
}

\begin{abstract}
The article is devoted to the methods which help to achieve precision of manufacturing long parts by means of mathematical modeling and dimensional analysis. The possibility of three-dimensional modeling of the shaft of the beater combine harvester "Vector" is considered and the model of turning of a long shaft is presented. The solution of problems arising in the manufacture of long shafts is possible by selecting the best way to install the work piece on the machine and calculate the optimal allowances of the work piece. Method of ad-justing the milling machine, using a special complex is introduced. For the analysis of technological process we will use the method of graph theory. Graph functional relationships established technological dimensional chains and is made up of the original equation. The solution of these equations allows us to find the optimal operating allowances and dimensions in the axial direction. To simplify and improve the calculations, we use the T-Flex CAD 3D complex module, translating the obtained formulas into a parametric table and linking the variables with the $3 \mathrm{D}$-model. Thus, we obtain a simulation dynamic model of dimensional connections of the technological process of manufacturing the shaft. Simulation of dimensional connections of the shaft manufacturing process in the radial direction also determined the operating allowances and dimensions. The proposed research methods are universal and can be applied to any details of this class.
\end{abstract}

\section{Introduction}

The difficulty in processing of machining parts such as long round bars is connected with the lack of rod's rigidity, what makes significant deformation of the part. In addition, the accuracy of processing depends on the initial deviations (curvature) of the work piece.

There are some common factors in detail processing such as installation error, insufficient rigidity of the machine, etc., affecting the quality of their production and specific ones, for example, insufficient rigidity of the part, causing deflection, which increases the system vibration, roughness of the treated surface; reduces the accuracy of the dimensions.

When technology of long parts manufacturing is developed, the influence of these factors should be excluded or minimized [1].

\footnotetext{
* Corresponding author: pme-dgtu@mail.ru
} 
Insufficient rigidity of the part can be compensated by selecting the optimal installation of the work piece on the machine, setting the optimal allowances and operating dimensions.

\section{Effect of the installation method on the deformation of long shafts during turning}

Let us consider the effect of the work piece installation on the accuracy of the long part processing on the example of the beater combine steel shaft "Vector" 45 (Fig. 1), length $1=$ $2099 \mathrm{~mm}$, diameter $\mathrm{D}=80 \mathrm{~mm}$.

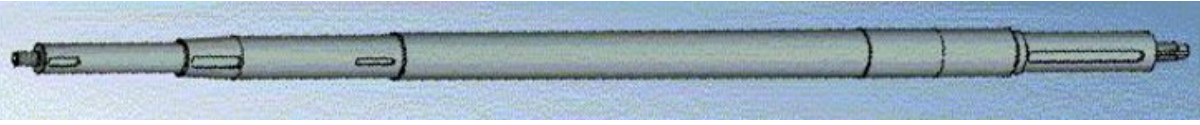

Fig. 1. The shaft of the beater combine harvester "Vector" (3D-model).

There are five ways to install the shaft on a lathe (Fig. 2):

Cantilever, holding one end of the work piece in the cartridge, and the second leaving free (Fig. 2, a);

One end of the work piece is clamped in the cartridge, the second is propped by the center, fixed in the tailstock of the machine (Fig. 2, b);

One end of the work piece is clamped in the chuck, the second is propped by the center, fixed in the tailstock of the machine, using a fixed backrest (Fig. 2, c);

One end of the work piece is clamped in the cartridge, the second is propped by the center, fixed in the tailstock of the machine, using a movable backrest (Fig. 2, d);

Both ends of the work piece can be installed in the centers using a movable backrest.

Using the spectral analysis method and T-FLEX CAD 3D environment, we will analyze the listed methods of shaft installation, identifying the advantages and disadvantages of each of them.

The first method of fixing (console). Advantages: easy to fix, no need to use the tailstock of the machine, expensive backrest and special adjustment to combine the axes of the chuck and tailstock quill.

The precision of the cantilever clamping during machining can be represented as the deviation of the part axis from the ideal straight line. Deviation $C_{z}$ (see Fig. 2, a) is a consequence of the impact on the work piece cutting force .

The deviation $C_{z}$ depends on the z coordinate, i.e. $C_{z}=f(z)$. The general equation of the axis deviation of the cylindrical work piece from the ideal line [2] has the following form:

$$
f(z)=\frac{c_{0}}{2}+\sum_{k=1}^{k} c_{k} \sin \frac{k \pi}{L} z
$$

Where $c_{0}, c_{1}$ - deviations of the work piece axis, respectively, at the origin and the current point; $L$ - the period of oscillation of the deviation values; $k$-the order number of the harmonic.

We assume that the period $L$ of oscillation coincides with the doubled length of the part: hence $L=2 l$. When $\mathrm{k}=1$ you will get the following:

$$
f_{1}(z)=c_{1} \sin \frac{\pi}{2 l} z
$$

The formula (2) shows that $f_{l}(z)$ increases from zero value at the end of the shaft fixed in the chuck $(z=0)$, to $c_{l}$ on the other end of the shaft $(z=l)$, i.e. the tilt axis.

In machining, such as turning, the main factor affecting the accuracy of processing is the radial component of the cutting force $P_{y}$ [3]. 
The calculated parametric module of the T-FLEX CAD 3D system was used to simulate the shaft axis deviations under the force $P_{y}$ action. To this end, a 3D-model of the shaft with superimposed force was created $P_{y}$.
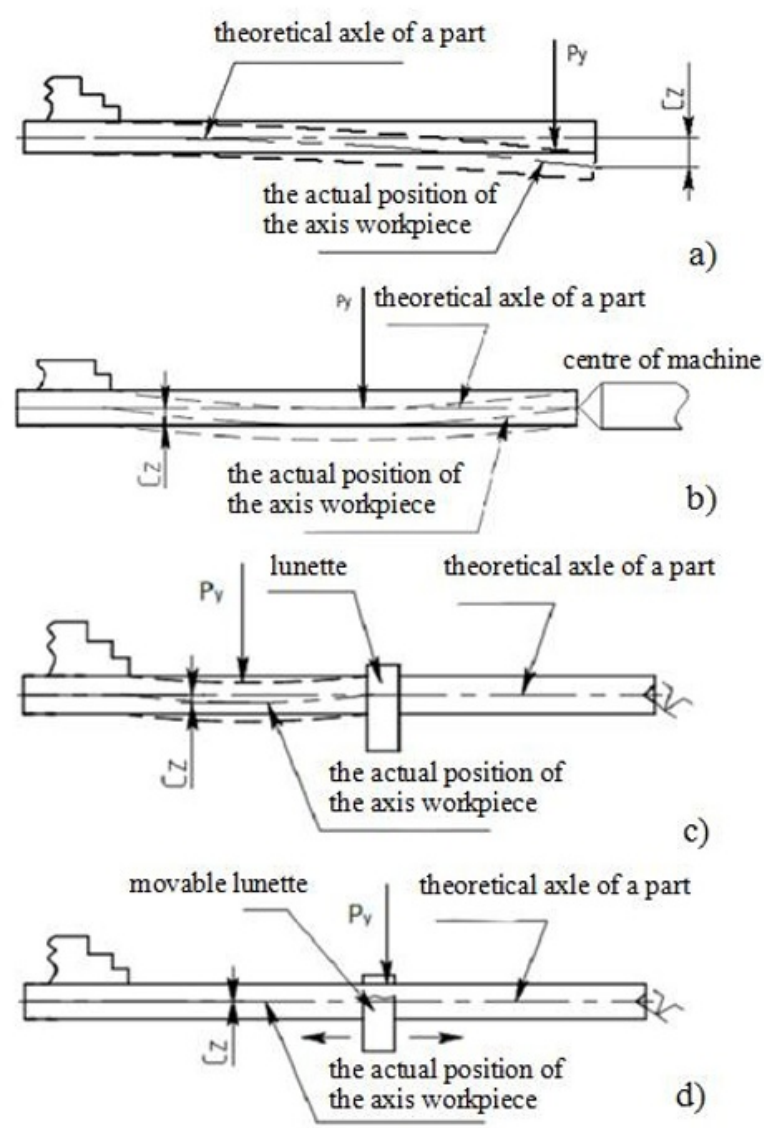

Fig. 2. The effects of cutting forces on the work piece: a) theoretical axle of a part; b) the actual position of the axis workpiece; c) lunette; d) movable lunette. 


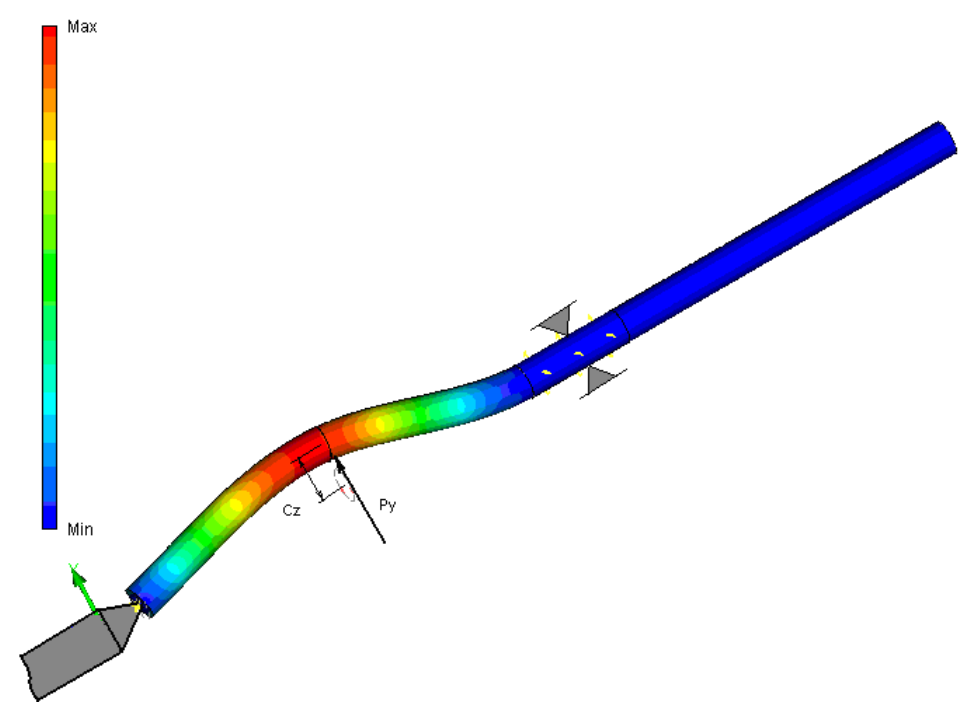

Fig. 3. Beater shaft using a fixed backrest and supporting center (3D-model).

Imitating the behavior of billet parts, enshrined in a three-chamber cartridge with the force of fixing to the surface in contact with cams of the cartridge. For making it, we split the 3D model of the shaft blank into a set of finite elements and apply the calculated force $P_{y}$, simulating the movement of the cutter. It can be seen that the deviation of the shaft axis depends on the $\mathrm{z}$ coordinate (Fig. 3). Therefore, with this deviation as a result of processing, we obtain not a cylindrical, but rather a conical surface. To exclude this, we will strengthen the rigidity of the technological system and consider the second method of installing the work piece.

The second method of installing the shaft blank (see Fig. 2, a) can be mathematically expressed from the general equation (1) of the axis deviation. The difference from the previous method is that when using the tailstock (supporting center), the distribution of deformation changes. The shaft axis deflection is sinusoidal, reaching a maximum in the middle of the shaft [9]:

$$
f_{2}(z)=c_{2} \sin \frac{\pi}{l} z
$$

This is confirmed by the 3D model of the work piece (Fig. 3). When fixing the work piece shaft, stiffness of the process system increases, we also increase the accuracy of the work piece dimensions, but the curvature of the shaft remains, leading to the barrel surface of the treated.

The advantages of this installation method: increasing the system rigidity, improving the quality of the machined surface of the work piece. Disadvantages: usage the backing center of the tailstock and more accurate adjustment of the machine, aimed at combining the axis of the supporting center and the chuck cams.

In order to eliminate the barrel shape of the shaft it is necessary to use a backrest.

The third method of installation of the work piece (see Fig. 2, в). Mathematical deviation of the shaft axis is described as well as the second method of the work piece installation, the expression (3). The only difference is that, since the stiffness of the shaft depends on the distance between its supports, $C_{z}$ will be not enough, therefore, there will be less deviation too.

Reducing a minimum barrel shape, using a movable backrest-the fourth method of installation of the work piece (see Fig. 2, d), in which the expression (3) is also correct, but 
the value $C_{z}$ is so small that it can be neglected, i.e. $C_{z}=0$, hence $f_{2}(z)=0$. Let's check it on the $3 \mathrm{D}$ model of the shaft blank. It can be seen that the deflection of the shaft blank is almost absent.

The advantages of this installation method of the work piece are obvious, but there are disadvantages as well - a very complexed adjustment, aimed at combining the axes of the cams, propping center and the backrest and the need to reinstall the work piece during processing, which leads to additional deviations.

To exclude the possible reinstallation if you install the billet fifth method, i.e. in the centres, using a movable bezel. However, there are difficulties which are associated with the transmission of torque to the work piece.

All of the above methods of the shaft blank installation have their advantages and disadvantages. The choice of installation is determined by the specific conditions of product manufacture.

Another important task that must be solved in the technology of long shafts manufacturing development is reducing the cost of the product, which is achieved by welldeveloped technology and optimal operating tolerances on the dimensions.

When modeling the accuracy of the shaft dimensions in the axial and radial directions and the operating dimensions, the process is important. So, the treatment process of the flail forage harvester shaft includes the following operations: milling machines, turning and keyway milling. The turning is performed in two settings: detailed part is set-up and the rest in set-up B.

For the analysis of technological process we will use the method of graph theory. The graph of dimensional connections of the process along the shaft axis is shown in Fig. 4.

Graph functional relationships established technological dimensional chains and is made up of the original equation.

The solution of these equations allows us to find the optimal operating allowances and dimensions in the axial direction $[4,5]$.

To simplify and improve the calculations, we use the T-FLEX CAD 3D complex module, translating the obtained formulas into a parametric table and linking the variables with the 3D-model. Thus, we obtain a simulation dynamic model of dimensional connections of the technological process of manufacturing the shaft.

The mathematical model allows you recalculate quickly all 126 equations, whether it is the design size $(A)$, technological $(\mathrm{S})$, or allowance $(\mathrm{Z})$ when this is recalculated to a $3 \mathrm{D}$ model of the part.

Simulation of dimensional connections of the shaft manufacturing process in the radial direction also determined the operating allowances and dimensions. 


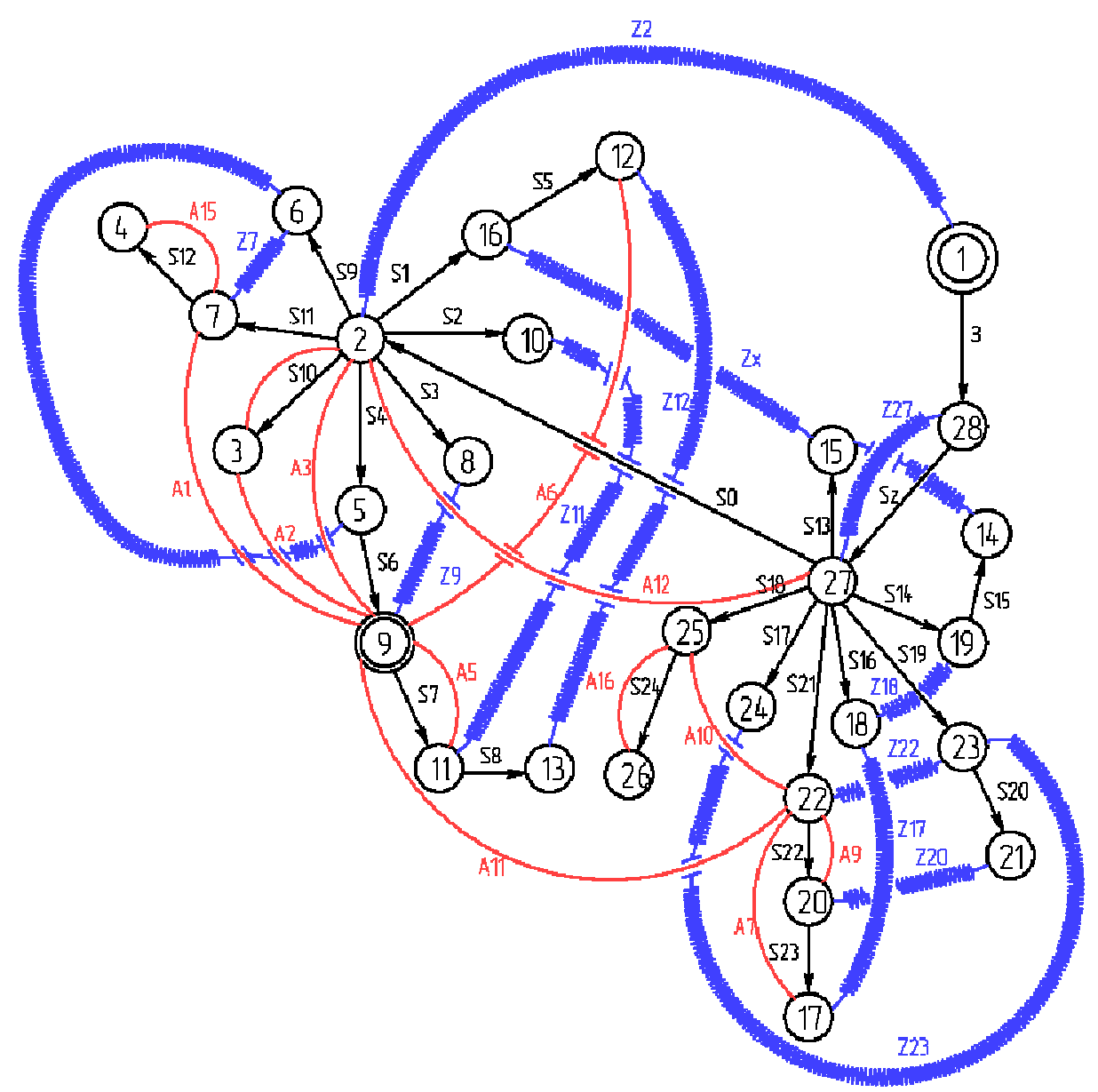

Fig. 4. Graph of technological process dimensional connections of manufacturing the beater shaft in the axial direction.

\section{Three-dimensional model of spatial deviations in the long shafts manufacture}

The accuracy of long shafts processing depends not only on the method of the work piece installation and dimensional connections of the technological system, but also on previous operations of the work piece preparation for processing, for example, straightening, performed to reduce the curvature of the work piece, which, in turn, affects the accuracy of the alignment of the shaft ends.

As a rule, long shafts are centered on milling and centering machines. Let's take a closer look at this operation. Installation of the work piece on the prism of the machine is carried out on the surface areas, which after processing serve as the main bases. From the analysis of the scheme of shaft installation on the milling-centering machine, it is seen that the work piece can be divided into three sections: the first - from the left end to the first prism; the second - between the prisms; the third - from the second prism to the right end.

The first and third sections are console anchors and can be described by an expression: 


$$
f(\varphi, z)=\frac{c_{0}(z)}{2}+\sum_{k=1}^{k=n} c_{k}(z) \cos \left(k \varphi+\varphi_{k}\right)
$$

where $\varphi$ - polar angle; z- axial coordinate [2];

The second section is described by the expression

$$
r_{(0)}=r_{0}+A_{0} \cos \Omega \varphi
$$

It is obvious that the deviation at some point of the shaft from straightness will inevitably lead to alignment error. But the expressions (4) and (5) reflect deviations are in only one plane. In fact, as it is shown by the measurement of deviations from the shaft axis of the work piece straightness, the deviation is both in the longitudinal and in the cross sections of the shaft.

For the analytical representation of the errors set of the shaft work piece in the axial and radial directions, we introduce a cylindrical coordinate system in which the deviation of the shaft axis is a function of two independent variables $\varphi$ and $z$.

In formula (4) the amplitudes $c_{0}(z)$ and $c_{k}(z)$ are not constant. They depend on the coordinate along the axis of the work piece.

The expression (4) shows what variable parameters are required to estimate the curvature of the shaft in three-dimensional space, by means of which you can create a 3D model of the shaft.

Using the T-FLEX CAD 3D system, we simulate the deviation of the real axis from the ideal one at five points $(\mathrm{R} 1 \div \mathrm{R} 5)$ with is a fixed distance between them and the polar angles of rotation $(\varphi 1 \div \varphi 5)$ of the maximum deviation relative to the selected coordinate system. The result of the expression analysis (4) is the calculated variable table in T-FLEX CAD 3D.

The calculation result can be represented as a cross-section profile. For clarity, the deviation was increased by 100 times. On the 3D model it is possible to carry out strength and dynamic analysis parts.

The angular position of the shaft cross-section profile is not constant. The sum of angular displacements leads to the surface waviness, which in turn can lead to irregularity of the removed allowance when processing the part, and the measurement error increases, which worsens the accuracy of the manufactured product. In [2] the profiles obtained under different conditions are considered in detail. The decisive factor affecting the shape of the part and its size is the ratio of the harmonic relative motion frequency $\lambda$ (part-tool) to the rotational speed of the $\mathrm{n}$ part during processing.

Thus, when $\lambda / \mathrm{n}=1$ we obtain the profile shown in Fig. 5, a. Retail parameters $\mathrm{R}$ and $\mathrm{r}$ are small, so we can assume that the shape deviations are insignificant, but there is an eccentricity equal to the amplitude A of the relative oscillations.

At $\lambda / n=2$, the profile is elliptical in nature (Fig. 5, b). At $\lambda / n=3$ it is a three-beam star (Fig. 5 , c). For integer values $\lambda / \mathrm{n}$, the profiles resemble stars with the number of rays equal to the multiplicity of this ratio, for fractional values $\lambda / \mathrm{n}$, there is a phase shift. 


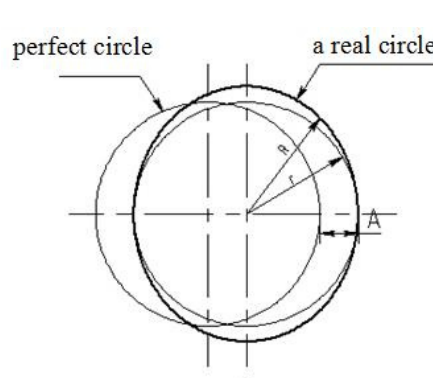

a)

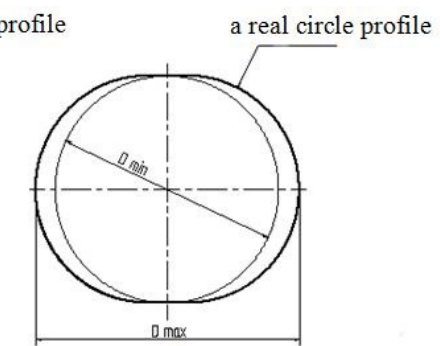

b)

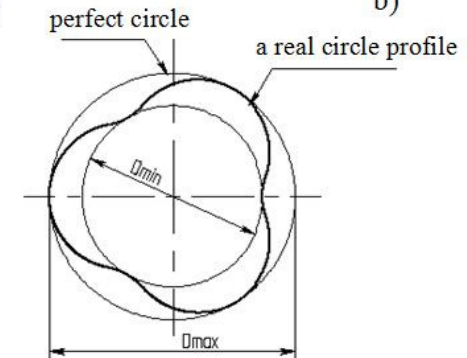

c)

Fig. 5. The calculated transverse profile of the shaft: a) $\lambda / \mathrm{n}=1$; б) $\lambda / \mathrm{n}=2$; в) $\lambda / \mathrm{n}=3$.

We derive General equations for circular and transverse waves [2]:

for zero waves: formula (5);

for the first wave:

$$
r_{(1)}=r_{0}+A_{0} \cos [\Omega(\varphi+1 \cdot 2 \pi)]
$$

for the second wave:

$$
r_{(2)}=r_{0}+A_{0} \cos [\Omega(\varphi+2 \cdot 2 \pi)]
$$

Thus, for the $p$-th wave:

$$
r_{(p)}=r_{0}+A_{0} \cos [\Omega(\varphi+p 2 \pi)]
$$

where $r_{0}$ - the radius of a geometrically correct cylindrical part; $A_{0}$ - the maximum amplitude of the oscillatory motion of the part-tool; $\varphi$ - rotation angle; $r_{(p)}$ - radius-vector of the point corresponding to the angle $\varphi_{n} ; A_{(p)}$ - the amplitude of the wave with the serial number $p ; \Omega$ - the ratio of the total angle of rotation of the part $(2 \pi)$ to the angle $\varphi$ rotation corresponding to the wavelength.

The expression (8) can be used to determine the deflection at any point of the shaft.

Let's make graphs of transverse and longitudinal waviness (Fig. 6, a and b) and transfer them to the environment T-FLEX CAD 3D. 


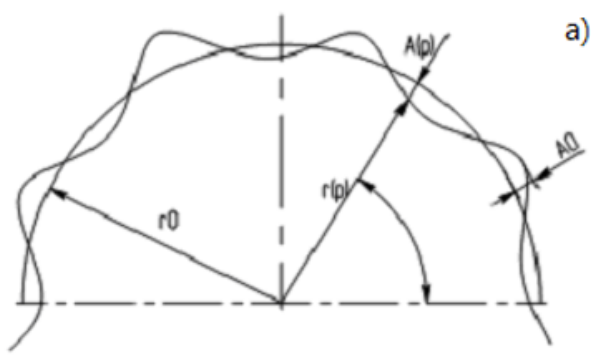

a)

b)

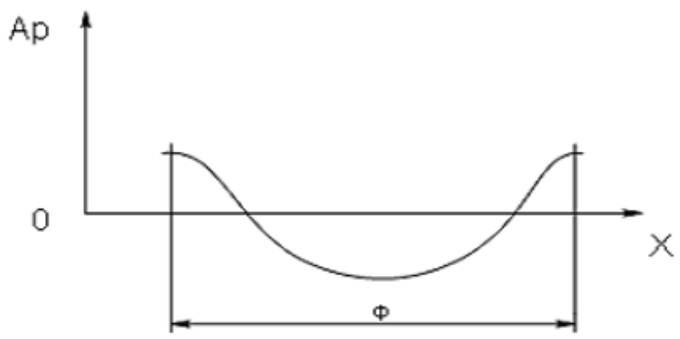

Fig. 6. Waves of shaft irregularities: a) in the transverse; b) in the longitudinal direction.

The possibility of three-dimensional modeling of the shaft is considered. We introduce real axis deviations $(\mathrm{R} 1 \div \mathrm{R} 5)$ and their polar angles $(\varphi 1 \div \varphi 5)$ into the table of variable parameters T-FLEX CAD 3D. For $\lambda / \mathrm{n}=3$ we obtained a 3D model. Since the shaft is considered on the milling-centering operation, the deviation in the second and fourth points are zero, since these points are the machine prisms, coaxially with the centering drill. Get the table of variable parameters in the environment T-FLEX CAD 3D and the corresponding 3D model. Given the fact that the rotation of the work piece cross-section profile is rotated around the axis completely through $140 \mathrm{~mm}$ shaft length, then at $1=2099$ $\mathrm{mm}$ we get that the profile will turn 15 times, i.e. $5400^{\circ}$. Therefore, at each controlled point the profile will be rotated by $1350^{\circ}$. The model of turning of a long shaft is presented. From it you can see when straightening the work piece shaft is not required, and when it is necessary.

\section{Conclusion. Improving the accuracy of adjustment of machines for the manufacture of long shafts}

Analysis of machines methods adjustment for the manufacture of long parts showed that the main problem is to ensure the alignment of the work piece axis and the installation axis of the machine elements. The axis of the long part is not a perfect straight line. Therefore, when adjusting, as a rule, a standard is used, the length of which is commensurate with the length of the part. In milling-centering operation deviation from the straightness of the standard leads to the fact that the axis of the mounting prisms will not coincide with the axis of the tool (see Fig. 6). The same is observed in the adjustment of lathes and other machines. More careful straightening and grinding of the standard lead to additional costs. In addition, this standard can not be used for adjustment of machines in the processing of other parts.

Therefore, a method for setting up the machine with a special complex was developed, which allows reducing the influence of errors in setting the standard, due to the use of a laser beam as an axis. 
Thus, the solution of problems arising in the manufacture of long shafts is possible by selecting the best way to install the work piece on the machine and calculate the optimal allowances of the work piece. The proposed research methods are universal and can be applied to any details of this class.

\section{References}

1. B.S. Balakshin, Mechanical Engineering. Fundamentals of mechanical engineering technology, Moscow (1969).

2. Yu.N. Landon, Mechanical Engineering. Functional interchangeability in engineering, Moscow (1967).

3. Handbook of mechanical engineer. In 2 t. T. 1 /Under the editorship of A.G. Cocilovo, R.K. Meshcheryakova. Mechanical Engineering, Moscow (1986).

4. I.S. Solonin, Mechanical Engineering. Calculation of Assembly and technological dimensional chains, Mocsow (1980).

5. M.E. Popov, Deforming processing of shafts: Publishing house of ISTU. Irkutsk (2013). 